\title{
Male Infertility and Klinefelter Syndrome (47, XXY)
}

\author{
Paul L Cummins*, Connor Harrity, David J Walsh and Kevin J Marron \\ Division of Reproductive Endocrinology \& Infertility, Sims IVF/The Sims Institute, Dublin, Ireland
}

\begin{abstract}
Klinefelter syndrome is a chromosomal condition that affects male physical and cognitive development. The symptoms associated with KS vary greatly and many males with KS are never diagnosed or treated. Affected individuals typically have small testes that do not produce as much testosterone as usual. Reduced testosterone can impact reproductive maturation greatly and can lead to delayed or incomplete puberty, breast enlargement (gynecomastia), reduced facial and body hair, and an inability to have biological children (infertility). Puberty is a time of rapid physical and psychological change, and hormone replacement treatment can successfully limit and reduce the symptoms of KS.
\end{abstract}

This case report encompasses a routine fertility evaluation in which a 38 year old Caucasian male was diagnosed with Klinefelter Syndrome (KS) and pronounced infertile. The case highlights the relative lack of awareness surrounding KS and the options which may enable KS males to produce healthy offspring.

Keywords: Male Infertility; Genetics; Klinefelter syndrome; 47XXY

\section{Background}

Klinefelter syndrome occurs in roughly 1:500-1:800 live births independent of race [1]. The syndrome stems from the acquisition of an additional $\mathrm{X}$ chromosome, a product of a nondisjunction event during meiosis [2] and is not taught to be hereditary. In male KS sufferers, testosterone production is minimal and leads to an abnormal body build. Phenotypically adult males will be tall, having a less muscular body, are often overweight with sparse facial and body hair, have larger breasts (gynaecomastia), weaker bones with broader hips. Also limited testosterone production during puberty often results in a small penis, testis and prostate gland. Additionally FSH and LH levels increase with age, excess of these hormones are known to cause causes hyalinization and fibrosis in the seminiferous tubules, where the sperm are normally located [3]. The majority ( $>60 \%)$ of cases are diagnosed post puberty when the symptoms are most obvious [4]. Men with KS often exhibit sexual dysfunction (impotence, lack of libido) and usually produce little or no sperm, with $>95 \%$ of cases being classified as clinically infertile [5]. Early identification and medical intervention of $47 \mathrm{XXY}$ males can greatly enhance physical, mental and emotional problems associated with the syndrome.

\section{Case Presentation}

A 38 year old Caucasian man attended our fertility clinic with his long term partner. They had been trying to conceive a child naturally for the past 3 years and had been unsuccessful to date with no biochemical pregnancy achieved. His past medical and social history were unremarkable. After routine endocrinology identified a hormone imbalance (Table 1) a presumptive diagnosis of hypogonadism was established. To evaluate gonadal function a full semen analysis (volume, count, motility and morphology) was ordered. Semen analysis results indicated azoospermia (absence of spermatozoa) and a repeat test 14 days later confirmed azoospermia. An investigatory genetic panel was then ordered (Karotype, CF mutation screen, Y deletion and CFTR Intron 8 Poly T Analysis) to evaluate any underlying genetic causes. All genetic tests were normal with the exception of Karyotyping which identified an additional $\mathrm{X}$ chromosome (47XXY vs. expected 46XY). This confirmed the diagnosis of KS which fulfilled criteria suspected during the latter ingestions.

\section{Discussion}

This report presents an adult male patient diagnosed with

\begin{tabular}{|l|c|c|}
\hline Assay & Value & Adult Male Reference Range* \\
\hline LH & 19.1 & $0.6-12.1 \mathrm{IU} / \mathrm{L}$ \\
\hline Prolactin & 457 & $69.0-417.0 \mathrm{mlU} / \mathrm{L}$ \\
\hline FSH & 30.7 & $1.0-12.0 \mathrm{mlU} / \mathrm{mL}$ \\
\hline Testosterone & 8.3 & $4.94-32.01 \mathrm{nmol} / \mathrm{L}$ \\
\hline
\end{tabular}

*All reference values were sourced from the abbott architect i1000sr automated immunoassay analyser.

Table 1: Patient's hormone values and expected reference ranges.

previously unsuspected Klinefelter syndrome as a result of cytogenetic testing for fertility investigations. This case report highlights the rarity of this syndrome and the increased awareness needed to treat KS in the early stages of puberty during which testosterone supplementation can lessen the physical and mental effects associated with KS. The major symptoms associated with KS are decreased intellect and an abnormal build which may not be obvious in the context of clinical manifestation of KS. Possibly the most abiding effect associated with KS is the reduced ability to produce offspring. However, with recent advances in assisted reproductive, 47XXY males have opportunities to avail of procedures which may enable them to produce offspring. With mixed variability and outcomes, TESE (Testicular Sperm Extraction) and ICSI (intracytoplasmic sperm injection) seem to be the most promising course of treatment providing that there are suitable numbers of sperm present, the sample can sometimes be frozen for use in future treatment cycles.

A study of 42 men with KS revealed that the sperm retrieval rate was $72 \%$ per testicular sperm extraction attempt, and 69\% (29 of 42 men) had adequate sperm found using Intracytoplasmic Sperm Injection (ICSI) [6]. A similar study retrospectively evaluated the micro-TESE/

*Corresponding author: Paul L Cummins, Division of Reproductive Endocrinology \& Infertility, Sims IVF/The Sims Institute, Dublin, Ireland, Tel: +353 (0) 1208 0710; Fax: +353 (0) 1208 0715; E-mail: paul.cummins@sims.ie

Received September 29, 2015; Accepted November 19, 2015; Published November 26,2015

Citation: Cummins PL, Harrity C, Walsh DJ, Marron KJ (2015) Male Infertility and Klinefelter Syndrome (47, XXY). J Clin Case Rep 5: 641. doi:10.4172/21657920.1000641

Copyright: (c) 2015 Cummins PL, et al. This is an open-access article distributed under the terms of the Creative Commons Attribution License, which permits unrestricted use, distribution, and reproduction in any medium, provided the original author and source are credited. 
ICSI performance in 134 patients with classic KS. Sperm retrieval rate by micro-TESE was $28.4 \%(38 / 134)$, the fertilization rate was $28 \%$ and the live birth rate per embryo transfer was $13 \%$ ( 5 children were born from 4 pregnancies) [7].

The patient described in this case study fulfilled the criteria for a standard couple in their late thirties seeking fertility treatment. After initial investigation the female partner's hormones presented normal while the male was indicative of hypogonadism. Semen analysis identified azoospermia and genetic investigations revealed the male carried a 47XXY karyotype. The couple was informed and was advised to undergo fertility counselling to discuss the options available. TESE was offered but declined in light of the patients high $\mathrm{FSH}$, age and the relatively high chance of genetic aberrations occurring in offspring. The couple have since sought fertility treatment using a sperm donor and intra-uterine insemination and have achieved a successful biochemical pregnancy.

Fertility treatment is an ongoing process and under the guidance of practitioners patients must constantly assess options available to them depending on their own reproductive capacity. Although intracytoplasmic sperm injection may be an option to some men with
KS it may not necessarily be successful, particularly if their associated partner has poor fertility prognosis.

\section{References}

1. Van Rijn S, Aleman A, Swaab H, Kahn R (2006) Klinefelter's syndrome (karyotype $47, \mathrm{XXY}$ ) and schizophrenia-spectrum pathology. Br J Psychiatry 189: $459-460$.

2. Wistuba J (2010) Animal models for Klinefelter's syndrome and their relevance for the clinic. Mol Hum Reprod 16: 375-385.

3. Pralea CE, Mihalache G (2007) [Importance of Klinefelter syndrome in the pathogenesis of male infertility]. Rev Med Chir Soc Med Nat lasi 111: 373-378.

4. Radicioni AF, De Marco E, Gianfrilli D, Granato S, Gandini L, et al. (2010) Strategies and advantages of early diagnosis in Klinefelter's syndrome. Mol Hum Reprod 16: 434-440.

5. Paduch DA, Fine RG, Bolyakov A, Kiper J (2008) New concepts in Klinefelter syndrome. Curr Opin Urol 18: 621-627.

6. Schiff JD, Palermo GD, Veeck LL, Goldstein M, Rosenwaks Z, et al. (2005) Success of testicular sperm extraction [corrected] and intracytoplasmic sperm injection in men with Klinefelter syndrome. J Clin Endocrinol Metab 90: 6263-6267.

7. Sabbaghian, M., et al., Comparison of sperm retrieval and intracytoplasmic sperm injection outcome in patients with and without Klinefelter syndrome. Urology, 2014. 83(1): p. 107-10. 